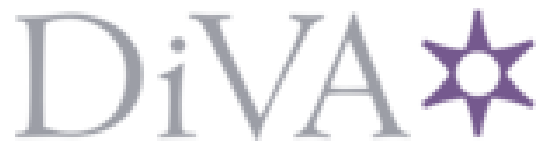

http://www.diva-portal.org

This is the published version of a paper presented at IEEE CBMS.

Citation for the original published paper:

Kane, B., Luz, S. (2009)

Assimilating Information and Offering a Medical Opinion in Remote and Co-located Meetings

In: 2009 22nd IEEE International Symposium on Computer-Based Medical Systems (pp. 475-480). IEEE

IEEE International Symposium on Computer-Based Medical Systems

N.B. When citing this work, cite the original published paper.

Permanent link to this version:

http://urn.kb.se/resolve?urn=urn:nbn:se:kau:diva-56973 


\title{
Assimilating Information and Offering a Medical Opinion in Remote and Co-located Meetings
}

\author{
Bridget Kane and Saturnino Luz \\ Department of Computer Science \\ Trinity College Dublin, Ireland
}

\begin{abstract}
Discussion on patient data, among hospital staff, plays an increasingly important role in inter-specialist communication. Effectiveness of a discussion depends, among other factors, on how well its participants perceive, assimilate and interpret information exchanged during a discussion. This paper reports a field study conducted to assess information assimilation among medical observer participants during PCDs in a hospital. Medically trained observer participants undertook a questionnaire at multi-disciplinary medical team meetings (MDTMs) in teleconference and colocated settings. Results show that participants are more likely to offer opinions in teleconference while their expectations on the long-term effects of treatment are more realistic in co-located PCDs than in teleconference PCDs. Surprisingly, the presentation of clinical findings, radiology and pathology is perceived to be clearer in teleconference, and respondents believe that they follow the discussion, know the patient management plan and understand the basis for decisions, better in teleconference than in colocated PCDs. While a higher educational value is attributed to teleconference PCDs, evidence suggests a trend to have more errors in teleconference, less critical evaluation and no expression of disagreement with patient management decisions made in teleconference.
\end{abstract}

\section{Introduction}

MDTMs, where patient cases are discussed, are common in hospitals and their practice is recommended in patient management guidelines, especially for cancer patients [7]. During a patient case discussion (PCD) specialists involved in a patient's care present their findings to peers (radiologists, pathologists and clinicians) and, following input from physicians, surgeons and oncologists, the patient's treatment is agreed in the circumstances. As well as patient management functions, PCDs serve professional development and educational purposes, especially in university teaching hospitals.

In order to elucidate the communication processes MDTMs we have conducted a detailed ethnographic study, reported elsewhere [4, 2]. Here we focus on non-vocal participants who attend MDTMs as part of their specialist training and with whom we conducted a targeted exercise that complements our published work on MDTMs. During a PCD, text summarising the patient's particulars is projected onto a side-wall in the room. Displayed information, e.g. name and smoking history, allows participants to check detail from time to time. PCDs open with a presentation of clinical findings, followed by a radiologist who shows imaging and a pathologist who illustrates any tissue sample. In teleconference, the main display is used to see remote participants (clinicians), unless an image is being broadcast from the main site (occupying the video channel). Contributions from clinical, radiology and pathology specialities allow for the patient's definitive disease staging to be determined [4]. PCDs also agree a management plan for the patient.

The first part of a PCD is characterised by the presentation of facts by individual specialists. The second part is much more interactive and less structured, as it deals with treatment options that involve more people and specialities. Information presented earlier may be reviewed in the light of later data. There is discussion and debate, as specialists interact and weigh evidence. When a decision is agreed, discussion moves to the next case. We use the term 'data capture' broadly to describe the perception, assimilation and interpretative processes people use when gathering information needed to perform tasks. Participants' ability to correctly perceive, assimilate and understand data presented to the meeting, through text, images and talk, is vital for MDTMs and the decision-making processes involved.

\section{The study}

Naturalistic observation, audio-visual recording and a questionnaire were used. A single page with 21 questions 
was designed to be completed during an individual PCD. Questions aimed to capture information retrieved through different types of cognitive processes used by an observer at a PCD, namely a) auditory attention and b) visual attention, c) interpretation of aural or visual data presented and d) evaluation, or judgement, on the decision process and benefit for the patient. Responses to questions on patient facts, professional opinion, the evaluation of peer contributions and the overall response rates are presented here. Analysis is conducted of the differences in responses in co-located and teleconference PCDs.

Three questions assess the direct capture of information presented either verbally, in text form, or via both modalities. These ask for information available in text displayed on the side wall and articulated in the PCD, namely the patient's name, age and smoking history. The date of birth is given in text as day, month, year and is articulated as years of age in talk. The correct answer requires either a calculation from the date displayed in text, or auditory attention to the age articulated in talk. Treatment plan questions (3), i.e. if a patient will have surgery, chemotherapy and/ or radiation therapy is determined from listening to verbal exchanges between participants in the latter part of a PCD.

Rating questions measured the level of a participant's understanding of the proceedings and were aimed directly at the pathology, radiology and clinical contributions and the underlying basis for the final decision. Other rating questions used 5-point Likert scales and sought the participant's level of agreement with decisions made and their opinion of the likelihood of a cure for the patient by the intervention proposed. This latter question assessed the participant's level of knowledge of the subject, i.e. their understanding of a patient's diagnosis, disease staging and treatment plan, and prior knowledge of the likely outcomes for such scenarios. Finally, participants were asked to assign an overall rating for the educational value for the PCD.

The exercise was reviewed by the lead clinician and validated before use. Video recordings were undertaken in conjunction with the exercise, to check data sources and correlate with responses. No senior member of the team participated in this exercise because of potential interference with their role, function or the business of the MDTM. All subjects in this exercise were observer participants, and qualified non-consultant hospital doctors. Individual responses were not tracked from week to week, because of anonymity agreed at the outset. For each PCD the video recording was reviewed and variations in the case presentation noted. For example, information might be presented in talk that differed from text on display or, data might be available on screen but omitted in talk. In 3 cases the patient's name was never articulated and in several cases 'age' was not mentioned. In some PCDs the verbal narrative started with the initial display of patient details and opened with: "This pa- tient presented to OPD with a history of ...", without the name or the age being articulated.

Examination of responses in conjunction with the review of video recordings provides information on the data source used in responding to questions. Where data are presented in both audio and visual forms and participants provide the correct answer, we cannot determine from which modality the information is captured. When information is given in a single form only (i.e. either audio or visual), or incorrect data are submitted, the response from the subject indicates the source from which they attempted to get information. For example, when a patient's year of birth is displayed on screen, but not articulated in talk, and the correct answer to the patient's age is submitted, we can assume that the year of birth is read from the visual display and the age in years is calculated. If incorrect data are articulated during the PCD, contradicting the correct data on visual display, the submitted response reveals from where the information probably emanated. Pearson's Chi-square test $\left(\chi^{2}\right)$ is used to check differences in categoric responses (correct, incorrect or non-responses). Scaled variables e.g. the number of answers per participant, are compared in different scenarios using $t$-tests. The Mann Whitney $U$ test is used to compare ratings. 'Don't know' responses were assessed as 'correct' or 'incorrect'. If the PCD made the information known, then 'don't know' is 'incorrect'; if the information was not made available, then a 'don't know' response is 'correct'.

\section{Results}

345 response sheets in total, from 92 PCDs are analysed. Mean completion rate per PCD is approximately 50\%. Of the 345 sheets, 85 were completed in 25 PCDs in teleconference and 260 completed in 67 co-located PCDs. Results are presented as follows: a) response rates (RR); b) matters of fact and interpretation; c) items that required retrieval from the dynamics of the PCD, i.e. the management decision; d) opinion on the decision, and e) rating of the educational value of the PCD. Differences for PCDs in teleconference versus co-located scenarios is monitored in the results.

Response Rates (RR) The response rate (RR) in teleconference is significantly higher than for co-located PCDs: $t$ test $=-8.15, d f=343, p<0.001$. It was thought that this difference might be accounted by the fact that PCDs in teleconference take longer than co-located PCDs [2]. However detailed analysis of RR patterns for different types of question reveals that other factors are also involved. Differences in RR for individual questions ranges from $-2.4 \%$ to $+28.2 \%$. RR is greater in teleconference in all except two cases: the patient's name and smoking history, two of the more personal questions in the exercise. The least difference in RR is to questions on facts that are displayed on the 
Table 1. \% of questions not answered in co-located and teleconference PCDs

\begin{tabular}{rrrcc}
\hline & Question & Teleconference & Co-located & Difference \\
\hline Facts from Text Display & Name & 2.4 & 0.8 & -1.6 \\
& Age & 2.4 & 13.5 & 11.1 \\
& Sex & 2.4 & 7.7 & 5.3 \\
Decisions Captured from Audio & Smoker & 27.1 & 24.6 & -2.5 \\
& Will patient have Chemotherapy? & 43.5 & 60 & 16.5 \\
& Will patient have Radiation therapy? & 51.8 & 65.4 & 17.2 \\
& Clinical & 34.1 & 70.8 & 19 \\
\cline { 2 - 4 } Evaluation of Presentation & Radiology & 25.9 & 62.3 & 28.2 \\
& Puestions on Belief & 36.5 & 33.8 & 7.9 \\
& Will patient be cured? & 64.7 & 46.5 & 10 \\
& Do you agree with decision? & 62.4 & 77.7 & 13 \\
& & & 77.3 & 14.9 \\
\hline
\end{tabular}

side-wall. The higher RR in teleconference is most marked for those questions that demand auditory attention. Table 1 shows the RR differences in co-located and teleconference PCDs on patient facts, opinion and management decisions. Patterns observed within each of the categories in Table 1, discussed in the following sections, suggest that there may be cognitive processes influencing how different types of information is captured, assimilated or interpreted in teleconference.

Matters of Fact and Interpretation Questions that ask of patient facts, namely 'forename', 'age', 'sex', and 'smoking status', are relatively well answered and have the highest RR. These facts are on display throughout the PCD in a text document and mentioned several times in talk. Table 1 shows that the RR for personal patient details, i.e. 'forename', 'age' and 'sex' are the same in teleconference, and the RR for these questions in co-located PCDs varies. Variation for 'age' is explained in the fact that the age must be calculated from the date of birth displayed on the side wall. This analysis shows that respondents use the audio source for data more often in teleconference.

Name: Respondents are generally able to answer this question. Instances (36) of phonetic name spellings suggest that audio is utilised for this data. Occurrences are more frequent in teleconference (3.5\% vs. $0.8 \%$ ) supporting the proposition that the audio source is accessed more frequently in teleconference. The patient's name was not articulated in 3 PCDs but was displayed in text form, and all respondents correctly provided those names. Thus, both audio and visual sources are used to capture this data in colocated and teleconference PCDs.

Age: No age was offered in 37 out of 345 responses, which were generated from 20 PCDs ( 2 from teleconference). For
PCDs where no age was submitted, all co-located, the patient's age was not articulated in talk. Response pattern suggests that the text display of the date of birth is the main source, and audio a secondary source, to determine the 'Age' in co-located PCDs and audio is the primary source in teleconference. There are significantly more responses for 'Age' in teleconference, but analysis for accuracy shows the error rate in teleconference is almost double that for co-located PCDs. $\chi^{2}$ is significant, $p<0.001$. Of the 308 'Age' responses, (225 co-located, 83 teleconference) over $80 \%$ of submissions were correct. In co-located PCDs, $84 \%$ are correct and $16 \%$ are incorrect. In teleconference PCDs by comparison, $69 \%$ of responses are correct but $31 \%$ are incorrect. The RR is much better in teleconference for 'Age': $97.6 \%$ vs. $39 \%$ from co-located PCDs.

Examination of the types of error, reveals the source used for information. Of the 63 'Age' errors, 43 are miscalculations from the written date of birth displayed on screen and the remaining 20 errors are in agreement with the age that was wrongly articulated in PCD talk. It is not possible to deduce whether the audio or text medium was preferred in all instances. For $66 \%$ of errors generated in teleconference, 'age' was incorrectly articulated across the interface and contradicted the date of birth on display. Audio is critical and participants say it is easier to hear in teleconference than at co-located PCDs. The speaker volume for sound from the remote sites is generally louder than speech in the same room. Microphones in the room capture and transmit sound to the remote site and the speaker reproduces the incoming audio signal only. Sex: The RR is better in teleconference. There is no significant difference in accuracy. Smoking history: RR is less in teleconference, accounted for by the fact that smoking history was articulated less frequently in teleconference PCDs. However, there are a significant number of false 'yes' errors (63\%) suggesting that 
observers to the PCD may not be fully attentive to the proceedings. The majority of patients discussed are either current, or ex-, smokers so we can deduce that the relatively high proportion of 'yes' errors represent guesses by the respondents (and a proportion of the correct responses are probably due to chance).

Patient Management Decisions In this section results on interpretations, especially of the patient management decision, are reported. It was asked if the patient will have a) chemotherapy, b) radiation therapy and /or c) surgery. To answer successfully, a respondent must attend to the conversation among the senior clinicians and follow the dynamics of the discussion. There is a greater cognitive demand on respondents in answering these questions, compared to questions on patient facts, as information is often interrogated, interpretations revised and options weighted. For example, it might be said: "So, we have a T1N0 here - we should proceed to lobectomy" which might elicit a response "I think she should be considered a T4 because ...". In another minute it might be agreed that more evidence is needed and to repeat the CT imaging. A surgeon might then add "his ECOG status is really poor ... even if he is a T1, I don't think he's fit for surgery anyway." Discussion might continue and a final decision made to have a PET scan and review the patient afterwards. Another scenario is for a team member to say "It looks like small cell, limited stage, disease, so you'll look after him", while nodding and making eye contact with the oncologist member of the team. For an observer to understand the management plan for chemotherapy agreed in this exchange, s/he will need to have followed the logic of the talk and have a knowledge of the roles of the respective members of the team. In most cases, the decision is summed up at the end of the PCD, so respondents potentially have more than one opportunity to retrieve the information needed. Although 3 questions that ask of the management plan for the patient are poorly answered, the RR is almost $20 \%$ greater in teleconference (Table 1). $\chi^{2}$ is significant, $p \leq 0.01$. The Mean correct response in teleconference is higher (1.5 vs. 0.9) than for colocated PCDs, but there is also a slight increase in the Mean errors in teleconference ( 0.44 vs. 0.36 co-located). Respondents' ability to capture data related to patient management from talk among the lead participants is much lower than their ability to capture patient facts. When the 'correct' responses for all patient facts and patient management decisions are examined, teleconference is found to be more productive overall: $\chi^{2}=18.1, d f=5, p \leq 0.003$.

Respondents' Opinions on Decisions Made Reported levels of agreement and understanding of the patient management decision is examined in 3 questions. Subjects are asked a) if they agree with the management strategy, b) if
Table 2. Attitudes towards decisions (\%)

\begin{tabular}{cccccc}
\hline \multicolumn{6}{l}{ Agree with decision? } \\
Scenario & Yes & Some & No & No Ans & $p<$ \\
\hline Coloc'd & 19.6 & 2.7 & 0.4 & 77.3 & .041 \\
Teleconf. & 34.1 & 3.5 & & 62.4 & \\
Understand the basis of decision? & & \\
Coloc'd & 18.1 & 4.6 & 0.8 & 76.5 & 0.011 \\
Teleconf. & 34.1 & 1.2 & 1.2 & 63.5 \\
Belief that the patient & might be cured as result of decision? \\
Scenario & Cor. & Accep. & Incor. & No Ans & \multirow{2}{*}{0.033} \\
Coloc'd & 12 & 5 & 15 & 78 & \\
Teleconf. & 24 & 4 & 7 & 65 \\
\cline { 1 - 5 }
\end{tabular}

they understand the basis of the decision and c) if s/he believes the patient will be cured as a result of the management plan agreed. Generally respondents did not answer c), unless they agreed with the management strategy (i.e. there was very little disagreement). The RR is greater for teleconference than for co-located PCDs (42\% vs. 25\%). Response pattern is similar for the 3 questions (Table 2). More responses are submitted in teleconference; respondents say they agree with, and understand the basis of the decisions, more often in teleconference. Respondents are also more confident in their belief that the decision might effect a cure for patients in teleconference PCDs. $\chi^{2}$ is significant for all 3 questions (Table 2).

'Rating' or Evaluation Questions When respondents rate the clarity of presentations and the educational value of a PCD, radiology rates highest and pathology second. Surprisingly clinical findings are rated least clear, even though the majority of respondents have more clinical expertise than either pathology or radiology. Both radiology and pathology utilise images to illustrate their professional opinion. Clinical findings were presented in monologues for this exercise. The measures given for radiology and pathology correlate with one another and with the amount of time these items were given during PCDs. There is no significant difference in rating scores for radiology and pathology in teleconference but the Mean rating for clarity is higher in co-located PCDs. These findings are consistent with the paradox observed in [1] between the need to see things while talking about them and the lack of demonstrated success when doing so in computer mediated environments. Combining presentation items, i.e. radiology, pathology and clinical findings, the overall rating of the presentations is significantly higher in teleconference $(p=0.005)$, but the statistical significance may be overstated given the high non-response rate.

PCDs in teleconference were expected to have more educational value from an observer's perspective. Contrary 


\section{Table 3. Responses on (a) patient facts and management decision questions, and (b) Ratio of correct responses in co-located and teleconference PCDs per response sheet}

(a)

\begin{tabular}{rccc} 
Questions & Correct & Incorrect & No Ans \\
\cline { 1 - 3 } Patient details & & & \\
teleconferall & 71 & 9 & 20 \\
co-located & 70 & 9 & 21
\end{tabular}

(b) Correct responses on Patient Decisions

Ratio

co-located

teleconference
4.18:1

$4.36: 1$

\begin{tabular}{|c|c|c|c|}
\hline \multirow[b]{2}{*}{ Questions } & \multicolumn{3}{|c|}{ Frequency \% } \\
\hline & Correct & Incorrect & No Ans \\
\hline \multicolumn{4}{|c|}{ Management Decision } \\
\hline overall & 23 & 8 & 69 \\
\hline teleconference & 33 & 10 & 58 \\
\hline co-located & 20 & 8 & 72 \\
\hline \multicolumn{4}{|c|}{ Correct responses on } \\
\hline \multicolumn{3}{|c|}{ Management Decisions } & Ratio \\
\hline \multirow{2}{*}{\multicolumn{3}{|c|}{ co-located }} & $0.41: 1$ \\
\hline & & teleconference & $0.61: 1$ \\
\hline
\end{tabular}

to expectations, teleconference PCDs have more mid-range scores than many co-located ones. Some co-located PCDs rate highly, but some score badly on educational value. Respondents give higher educational ranking when patient findings are clearly presented $(p \leq 0.01)$. Overall, $51 \%$ of PCDs in teleconference and $63 \%$ of co-located PCDs rate in the top half of the scale. Table 3 (a) and (b) summarises the results on patient facts and management decisions. Patient 'facts' retrieved from the first part of the meeting, are formally presented in text and/or talk. The management decision must be retrieved from conversation among the lead participants. Tables 3 (a) and (b) show that the rate of acceptable and correct responses is low when answers need to be retrieved from conversation (and the non-response rate is high). Results also show that in co-located PCDs respondents tend not to submit any response, rather than submit an error for all questions. Overall error rates for patient details and management decision is about the same $(8-9 \%$ of questions).

\section{Conclusions}

Respondents are generally able to correctly capture information, particularly for facts on display (Table 1) but it is difficult to assimilate data from conversation (Table 3). Results for factual data suggest that people are more audioattentive than visual-attentive in teleconference, and are more inclined to retrieve information from talk rather than text in teleconference. Overall efficiency is increased in teleconference (i.e. more correct responses), but there is also a greater proportion of errors. At co-located PCDs efficiency is maintained (i.e. a high correct rate) at the expense of RR (lower). Respondents are more conservative in co-located PCDs and likely not to submit an answer than submit an incorrect response. Both audio and visual data sources are used and response rates are improved, overall, in teleconference PCDs. It is useful to consider the responses in the context of a) direct capture of data explicitly presented versus b) data captured from conversation and interaction among the active participants, and c) examine how cognitive processes may be impacted by teleconferencing, evidenced in responses to questions on opinion and belief.

Use of Audio: Audio is shown to be the most important source for both factual and decision data, particularly in teleconference. The preferred use of audio is evidenced in the analysis on factual data, especially errors for 'Age'. Phonetic spelling of names suggest that audio is used. But instances of correct name identification when it was not articulated show that audio was not the only source. For 'Age', which is presented in both text and talk, there are significantly more errors in teleconference. Analysis of responses in conjunction with the audio-visual recordings show that respondents rely more on audio than visual text data for information in teleconference. The ability to gather data on patient management decisions, from listening to the conversation among the lead participants in teleconference, is also evidence to the importance of audio at meetings. Colocated PCD observers are particularly weak at reporting on the patient management plan. Audio is reported clearer in teleconference, which may also contribute to the audio source being preferred over visual data in teleconference. The exercise itself may also have had an effect of influencing respondents to rely more on the audio channel (since they were engaged in writing an answer on the response sheet). These results suggest that support for better audio at co-located meetings will improve MDTM efficiency, and reinforce the need for high quality audio in teleconference. Use of Visual data: These results support those of [9] who highlights the need to support talk about objects. We show that optimising the display of visual data improves performance, even in teleconference. We further bring attention to the complimentary issue of the need to support 'peripheral objects', i.e. objects that are not necessarily the focus of talk but nevertheless contribute information that provides 
important context for the talk/discussion. The text display of information during a PCD is evidently used by respondents. Given a choice of what to view, participants at meetings demonstrate a need to be able to see the people at the remote site. Yet the results here demonstrate that the capture of factual information is more efficiently achieved through text, which makes an argument for having multiple screens available in teleconference PCDs. We show that when factual data are presented in competing text and audio forms there is a bias towards the audio source and a consequent greater potential for error. An argument can be made for the need for three screens, or three dedicated visual channels as follows. a) Persistent text display of pertinent patient facts would serve to clarify important identifiers and details and also provide an awareness mechanism to know which patient is being discussed. b) Face-to-face view with the remote party, considered important by participants at MDTMs, may contribute to the slightly greater efficiency in capturing information from conversation in teleconference, since team members tend to sit facing the screen displays during meetings. c) The third screen could display the image artefact being discussed, such as the radiological image. Teleconference Issues: The MDTM is a loosely coupled work setting with relatively high common ground and readiness for collaboration, and can be expected to succeed in working in teleconference [8]. However, there remain issues with teleconferencing that are poorly understood and require further research. Respondents in this exercise are more willing to offer opinions for almost all questions in teleconference, and they are almost 5 times more likely to offer their opinion on data captured from talk in teleconference PCDs compared with co-located PCDs. It is unclear why respondents more willingly submit answers, particularly incorrect responses in teleconference PCDs. People seem to hold more positive attitudes in teleconference since no disagreement is expressed in these results (Table 2). This positive attitude is most noticeable in the belief that the patient will be 'cured' in PCDs in teleconference, (seen in the slight increase in errors to this question in teleconference). It is surprising that there is an increase (even if small) in the number of errors submitted for teleconference PCDs to the question on belief of cure for the patient since the chance of 'cure' is remote. Audit of these patients shows that the Median survival is 8 months, 1 - and 3-year survival is $40 \%$ and $20 \%$ respectively. There is no clinical reason why patients discussed in teleconference might expect to have an improved outcome over patients discussed in a co-located PCD. The presentation of all findings rates slightly better in teleconference, but the overall educational rating is less than expected. The preference for co-located PCDs is also reflected in other evaluations reported in $[3,6]$.

In conclusion, observers at meetings find it difficult to capture information from talk, and we show that commu- nication through the use of image artefacts, such as radiology and pathology, improves the clarity of the communication. For information to be successfully captured from dialogues, it needs to be explicitly articulated in talk or supplemented with clear visual data. Teleconferencing has engaged substantial CSCW research in the past [5]. However, some issues arise when this technology is employed at MDTMs are still not fully understood. Why, for instance, are observer medical experts more optimistic and more willing to offer opinion in teleconference? Why do teleconference PCDs appear more successful at conveying information, even though video-mediated interaction is perceived as less satisfying than co-located discussion by the participants? Efforts to support synchronous communication have tended to concentrate on 'same time, different place' issues. Although further research is needed to answer these questions, a need is identified to better support 'same time, same place' co-located dialogue as well as methods to evaluate medical decision-making in teleconference.

\section{Acknowledgments}

Our thanks to the respiratory MDT in St James's Hospital, Dublin, for their generous co-operation. This research is supported by the SFI Research Frontiers grant under the National Development Plan.

\section{References}

[1] S. Bly. Talking about talking about things. Human-Computer Interaction, 18:181 - 191, 2003.

[2] B. Kane and S. Luz. Multidisciplinary medical team meetings: An analysis of collaborative working with special attention to timing and teleconferencing. Computer Supported Cooperative Work (CSCW), 15(5-6):501 - 535, December 2006.

[3] B. Kane and S. Luz. Probing the use and value of video for multi-disciplinary medical teams in teleconference. In Proceedings of the 19th IEEE International Symposium on Computer-Based Medical Systems, pages 518-523. IEEE Computer Society, July 2006.

[4] B. Kane and S. Luz. Achieving diagnosis by consensus. Computer Supported Co-operative Work (CSCW), on-line, April 2009. DOI:10.1007/s10606-009-9094-y.

[5] A. J. S. Kathleen E. Finn and S. B. Wilbur, editors. VideoMediated Communication. Lawrence Erlbaum Associates, Inc., 1997.

[6] E. J. Macaskill, S. Thrush, E. M. Walker, and J. M. Dixon. Surgeons' views on multi-disciplinary breast meetings. European Journal of Cancer, 42:905-908, 2006.

[7] NCCAC. The diagnosis and treatment of lung cancer, volume CG24. NICE, February 2005.

[8] J. S. Olson, G. M. Olson, and D. Meader. Distance matters. In A. J. S. Kathleen E. Finn and S. B. Wilbur, editors, VideoMediated Communication, chapter Face-to-Face Group Work compared to Remote Group Work with and without Video. ACM Press, 2000.

[9] S. Whittaker. Things to talk about when talking about things. Human-Computer Interaction, 18:149 - 170, 2003. 